\title{
Enhancement of Transgene Expression by NF-Y and CTCF
}

\author{
Devon Zimmerman ${ }^{\mathrm{a}}$, Krupa Patel ${ }^{\mathrm{a}}$, Matthew Hall ${ }^{\mathrm{a}}$, \& Jacob Elmer ${ }^{\mathrm{a}}$
}

\author{
${ }^{a}$ Department of Chemical Engineering \\ Villanova University \\ 800 East Lancaster Avenue \\ Villanova, PA, USA 19085 \\ *Corresponding Author: \\ Dr. Jacob Elmer \\ 119 White Hall \\ Department of Chemical Engineering \\ 800 East Lancaster Avenue \\ Villanova, PA 19085 \\ jacob.elmer@villanova.edu \\ (610) 519-3093 (P) \\ (610) 519-5859 (F)
}




\begin{abstract}
If a transgene is effectively delivered to a cell, its expression may still be limited by epigenetic mechanisms that silence the transgene. Indeed, once the transgene reaches the nucleus, it may be bound by histone proteins and condensed into heterochromatin or associated with repressor proteins that block transcription. In this study, we sought to enhance transgene expression by adding binding motifs for several different epigenetic enzymes either upstream or downstream of two promoters (CMV and EF1 $\alpha$ ). Screening these plasmids revealed that luciferase expression was enhanced 10 -fold by the addition of a CCAAT box just upstream of the EF1 $\alpha$ promoter to recruit nuclear transcription factor Y (NF-Y), while inserting a CCCTCbinding factor (CTCF) motif downstream of the EF1 $\alpha$ promoter enhanced expression 14-fold $(14.03 \pm 6.54)$. ChIP assays confirmed that NF-Y and CTCF bound to the motifs that were added to each plasmid, but the presence of NF-Y and CTCF did not significantly affect the levels of histone acetylation $(\mathrm{H} 3 \mathrm{~K} 9 \mathrm{ac})$. Overall, these result show that transgene expression from the EF1 $\alpha$ promoter can be significantly increased with motifs that recruit NF-Y or CTCF.
\end{abstract}




\section{Introduction}

Condensation of DNA by histone proteins within the nucleus allows the cell to maintain a large amount of DNA ( $\sim 6$ billion bp) within a relatively small nucleus (1-10 $\mu \mathrm{m})$, but it can also sequester genes into inaccessible regions of chromatin (i.e. heterochromatin) that are transcriptionally inactive. However, specific post-translational modifications of histone residues can activate genes by either relaxing the local chromatin structure or recruiting transcription factors that enhance gene expression. ${ }^{1,2}$ For example, acetylation of the amine on lysine 9 of histone 3 ( $\mathrm{H} 3 \mathrm{~K} 9 \mathrm{ac})$ activates genes by weakening the electrostatic interactions between the histone and DNA. In addition to acetylation, there are several other histone modifications and specific combinations thereof that can either activate or silence genes. Indeed, while $\mathrm{H} 3 \mathrm{~K} 9 \mathrm{ac}$ is associated with transcriptional activation, trimethylation of that same residue $(\mathrm{H} 3 \mathrm{~K} 9 \mathrm{me} 3)$ is associated with transcriptional inactivation. ${ }^{3}$ Altogether, these mechanisms that control the transitions between tightly packed heterochromatin and relaxed euchromatin to control gene expression are known as epigenetics.

In addition to regulating host cell gene expression, epigenetic modifications can also defend the cell by silencing foreign viral genes. ${ }^{4}$ Unfortunately, both viral and non-viral gene therapy treatments can be hindered by these epigenetic defenses as well. For example, transgenes delivered via adenovirus, lentivirus, and $\gamma$-retrovirus have been silenced through methylation of the viral promoter ${ }^{5,6}$ and histone tail modifications (e.g. deacetylation of $\mathrm{H} 3$ and $\mathrm{H} 4{ }^{7}$ and/or the conversion of $\mathrm{H} 3 \mathrm{~K} 9 \mathrm{ac}$ to $\mathrm{H} 3 \mathrm{~K} 9 \mathrm{me} 2^{8}$ ). Plasmid DNA delivered with non-viral vehicles can also be silenced by several different histone modifications, including $\mathrm{H} 3 \mathrm{~K} 9 \mathrm{me} 2$, H3K9me3, H4K20me2, H4K20me3, and H3K27me3. ${ }^{9-11}$ Altogether, these previous studies show that epigenetic silencing mechanisms must be considered when performing gene delivery experiments.

A few strategies have been developed to reduce transgene silencing, including the inhibition of key epigenetic enzymes. For example, inhibition of lysine specific demethylase 1 (LSD1) with SL11144 can increase methylation of H3K4, decrease methylation of H3K9, and demethylate DNA methyltransferase 1 (DNMT1) to globally reduce DNA methylation and prevent gene silencing. ${ }^{12,13}$ Many DNMT inhibitors such as 5-Aza-2'-deoxycytidine and zebularine have been used to prevent methylation of DNA. ${ }^{14,15}$ Several inhibitors of histone deacetylases (HDACs) have also been shown to increase transgene expression, including Entinostat (a HDAC $1 / 3$ inhibitor) ${ }^{16}$, Tubacin (HDAC6i) ${ }^{17}$, Trichostatin A (HDAC6i) ${ }^{18-20}$, and Vorinostat (pan-HDACi) ${ }^{21-23}$. However, it is important to note that some of these HDAC inhibitors enhance transgene expression by influencing cytoplasmic transport instead of directly influencing histone modifications.

As an alternative to small molecule inhibitors, Kay et al. modified the sequence of the plasmid itself to avoid epigenetic silencing in a more localized fashion. Specifically, since the antibiotic resistance gene and origin of replication usually lie dormant within eukaryotic cells and serve as a nucleation point for heterochromatin formation that eventually spreads to the transgene, those elements were moved into an intron that is continuously transcribed. The resulting mini-intronic plasmid (MIP) significantly increases both the magnitude and duration of transgene expression in vitro and in vivo. ${ }^{11,24,25}$ In addition, since promoter methylation can lead 
to gene silencing, ${ }^{26,27}$ removal of $\mathrm{CpG}$ motifs within CMV promoters has also been shown to provide higher long-term transgene expression. $^{28}$

The goal of this work was to prevent transgene silencing by adding motifs for a variety of proteins that are known to epigenetically regulate gene expression. For example, a CCAAT motif was inserted upstream and downstream of two promoters (EF1 $\alpha$ and CMV) to recruit nuclear factor $\mathrm{Y}(\mathrm{NF}-\mathrm{Y})$, a histone-fold domain protein that is associated with transcriptional activation. $^{29}$ Additional transcription factor binding sites (TFBS) that were inserted into the plasmid recruit other enzymes that provide a variety of modifications, including histone acetylation $\left(\mathrm{ATF} 2^{30}, \mathrm{SP} 1^{31}, \mathrm{HNF}^{32}, \mathrm{Zta}^{29}, \mathrm{POU} 6 \mathrm{~F} 1^{33}, \mathrm{VSX}^{33}\right.$ ), histone methylation (AP-1 ${ }^{34}$ and $\mathrm{AP}-2 \mathrm{a}^{34}, \mathrm{MYB}^{34}, \mathrm{NF}-\mathrm{Y}^{34}$, GFY-Staf ${ }^{34}$, Ying-Yang ${ }^{35}$ ), and general chromatin remodeling (Androgen Receptor ${ }^{36}, \mathrm{CTCF}^{35}$, Poly $(\mathrm{dA}: \mathrm{dT})^{37}$, FOXA1 ${ }^{38}$ ). Overall, we show that adding NF-Y and CTCF motifs can significantly improve transgene expression, but in a location and promoterspecific fashion.

\section{Materials and Methods}

\section{Preparation of Plasmids}

The pEF-Luc plasmid was constructed by cloning a luciferase gene into the pEF-GFP plasmid (Plasmid \#11154, Addgene, Cambridge, MA). The pCMV-Luc plasmid was then created by replacing the EF1 $\alpha$ promoter with the CMV promoter. The sequence of each promoter and the corresponding plasmid backbone can be found in Figures S1, S2, and S3.

Each of the motifs shown in Table S1 were inserted 19 bp upstream of each promoter (at position -753 relative to the TSS of CMV and -222 for EF1 $\alpha$ ) using site directed mutagenesis, while the downstream motifs were inserted via oligo annealing cloning between EcoRI and KpnI sites (at position +13 relative to the TSS of CMV and +1194 for EF1 $\alpha$, see Figure 1). The sequence of each inserted motif, along with the corresponding promoter and reporter gene, was verified via Sanger sequencing.

\section{Cell Transfections and Luciferase Assays}

Human prostate cancer cells (PC-3) were seeded on 24 well plates at a density of 50,000 cells/well in fetal bovine serum-containing media (SCM) - Gibco® RPMI 1640 (ThermoFisher Scientific Inc., Waltham, MA) - 24 hours prior to transfection. Polyplexes were prepared by mixing branched PEI (MW=25,000, Sigma Aldrich, St. Louis, MO) and the expression plasmid(s) in a 1:1 w/w ratio (8:1 N:P) with a total of $200 \mathrm{ng}$ DNA/well, then incubating the mixture for 20 minutes at room temperature. Meanwhile, the SCM in each well was aspirated and replaced with serum-free media (SFM). Polyplexes were simultaneously added to each well and cells were then incubated for an additional 6 hours at $37^{\circ} \mathrm{C}$ in SFM, after which time the media was exchanged again with fresh SCM. Alternatively, to demonstrate the ability of the motifs to enhance transgene expression independently of the vehicle used (Figure 2) and ensure higher signals for ChIP assays (Figure 5), Lipofectamine LTX with PLUS reagent (Thermo Fisher, Hercules, CA) was used to transfect cells according to the manufacturer's protocol. Finally, the data in Figures S5 and 1 were obtained by transfecting cells with jetPEI (Polyplustransfection ${ }^{\circledR}$, Illkirch, France) in a 5:1 nitrogen:phosphate (N:P) ratio. 
At 48 hours post-transfection, luciferase expression was measured using a Luciferase Assay kit (Promega, Madison, WI). Raw luminescence values for each triplicate were averaged and then normalized to the luminescence of the corresponding pEF-Luc or pCMV-Luc control without inserted motifs to obtain the relative luminescence values shown in each figure.

\section{Transient ChIP and qPCR Analysis}

PC-3 cells were grown in T-75 flasks to $\sim 50-70 \%$ confluency, transfected with $1.5 \mu \mathrm{g}$ of plasmid DNA using Lipofectamine ${ }^{\circledR}$ LTX with PLUS ${ }^{\mathrm{TM}}$ Reagent (ThermoFisher Scientific Inc., Waltham, MA), and then incubated for 48 hours at $37^{\circ} \mathrm{C}$. Cells were then trypsinized to detach them from the flask and counted on a hemocytometer to determine their concentration. A sample of approximately $1-5 \times 10^{6}$ cells was then treated with formaldehyde ( $1 \%$ final concentration) at room temperature for 10 minutes to cross-link the genomic DNA (including plasmids) to any bound proteins. Input samples of the genomic DNA were set aside for qPCR analysis, then chromatin immunoprecipitation was performed according to the manufacturer's instructions for the SimpleChIP® Enzymatic Chromatin IP Kit (\#9003, Cell Signaling Technology, Danvers, MA) with minor alterations. Specifically, cells were lysed via sonication with four 20 -second pulses at $10 \%$ power with a 1 minute incubation between pulses with a Branson Digital Sonifier and a Branson Model 102C Probe. With the exception of the NF-YA antibody (Abcam, \#ab139402), all antibodies used for immunoprecipitations were obtained from Cell Signaling Technologies (\#3418S anti-CTCF Ab, \#9649S anti- H3K9ac Ab; IgG \& anti-H3 antibodies were included in the SimpleChIP® kit). Threshold cycle $\left(\mathrm{C}_{\mathrm{T}}\right)$ values were then measured for each sample using plasmid-specific primers and SYBR ${ }^{\mathrm{TM}}$ Select Master Mix for CFX (ThermoFisher Scientific Inc., Waltham, MA) on a QuantStudio3 Real Time PCR System. The percent input (\%input) for each sample was then calculated with Equation 1.

$$
\% \text { Input }=2 \% * 2^{\left(C_{T, i n p u t}-C_{T, I P} \text { sample }\right)}
$$

Eqn. 1

\section{Plasmid Copy Number}

The fold-change in nuclear uptake of lead enhancer plasmids relative to the control plasmid was quantified by determining the copy number of transfected pDNA relative to the actively transcribed housekeeping gene RPL30. qPCR with SYBR $®$ Green was performed using $2 \mu \mathrm{l}$ SimpleChIP® Human RPL30 Exon 3 Primers from Cell Signaling Technology and $1 \mu \mathrm{l}$ each of forward and reverse primers specific to the pDNA enhancer sequence. Relative copy number was determined using the $2^{\Delta \mathrm{Ct}}$ method where $\Delta \mathrm{C}_{\mathrm{t}}$ is the difference in $\mathrm{C}_{\mathrm{t}}$ between reactions performed with RPL30 primers and enhancer primers. The fold-change in copy number was determined using the $2^{\Delta \Delta \mathrm{Ct}}$ method in which $\Delta \Delta \mathrm{C}_{\mathrm{t}}$ is the difference in $\Delta \mathrm{C}_{\mathrm{t}}$ between the sample plasmids and the control plasmid. 


\section{Statistics}

Statistical significance was evaluated in $\mathrm{R}$ studio using Analysis of Variation testing (ANOVA). For all experiments, a 95\% confidence interval test was used to determine if data was statistically significant $(\mathrm{p}<0.05)$.

\section{Results and Discussion}

\section{Effects of Motifs on Transgene Expression}

Of the 14 motifs tested, only a few significantly enhanced transgene expression (Figure 1; see Table S1 for numerical values). Some of the strongest enhancement was observed with the NF-Y motif (TCAGCCAATCAGCGAG), which significantly increased transgene (luciferase) expression when inserted upstream of the EF1 $\alpha$ promoter (10.4 \pm 5.8 -fold). Inserting the NF-Y motif downstream of the CMV promoter also seemed to increase transgene expression about 4-fold, but the effect was not statistically significant. Likewise, no enhancement was observed when the NF-Y motif was inserted upstream of the CMV promoter. This lack of enhancement of the CMV promoter may be due to the fact that eight separate NF-Y motifs (CCAAT or ATTGG, see Figure S2) are distributed throughout the native CMV promoter, such that an additional CCAAT site may be redundant. In contrast, the EF1 $\alpha$ promoter does not contain any CCAAT motifs.

Strong enhancement $(14.0 \pm 6.5$-fold $)$ was also observed when the motif for CCCTCbinding factor (CTCF, AGACCACCAGAGGGCACCA) was inserted downstream of the EF1 $\alpha$ promoter. As was the case for the NF-Y motif, the CTCF motif did not enhance expression from the CMV promoter. Interestingly, further analysis revealed that the CMV promoter already contains a high-affinity CTCF motif (Figure S4), while the EF1 $\alpha$ promoter contains only a partial low-affinity CTCF motif. It is also worth mentioning that the plasmid backbone itself contains three high-affinity CTCF sites in the PolyA region and another high-affinity CTCF site just upstream of the EF1 $\alpha / C M V$ promoters (Figure S4). Therefore, an additional CTCF site may be redundant in the CMV promoter and upstream of the EF1 $\alpha$ promoter, but beneficial when inserted downstream of the EF1 $\alpha$ promoter.

It is also interesting to note that a directly comparison of the two native promoters shows that the CMV promoter (which contains NF-Y and CTCF motifs) consistently provides 4-fold higher transgene expression than the EF1 $\alpha$ promoter (Figure S7). However, as shown in Figure 1, adding either motif to the EF1 $\alpha$ promoter provides 2-3 times more transgene expression than the CMV promoter. We also attempted to further enhance expression by creating an EF1 $\alpha$ promoter with both an upstream NF-Y motif and a downstream CTCF motif, but the combination of motifs did not significantly increase luciferase expression relative to an EF1 $\alpha$ promoter with a single NF-Y motif. (Figure S5). 
In contrast to NF-Y and CTCF, some of the motifs significantly decreased transgene expression when inserted downstream of the promoters. For example, inserting the GFY-Staf motif downstream of both promoters decreased luciferase expression by $60-80 \%$, while several other motifs (POU6F1, AP-1, AP-2a, Sp1, and Zta) significantly decreased expression (30-80\%) from the CMV promoter.
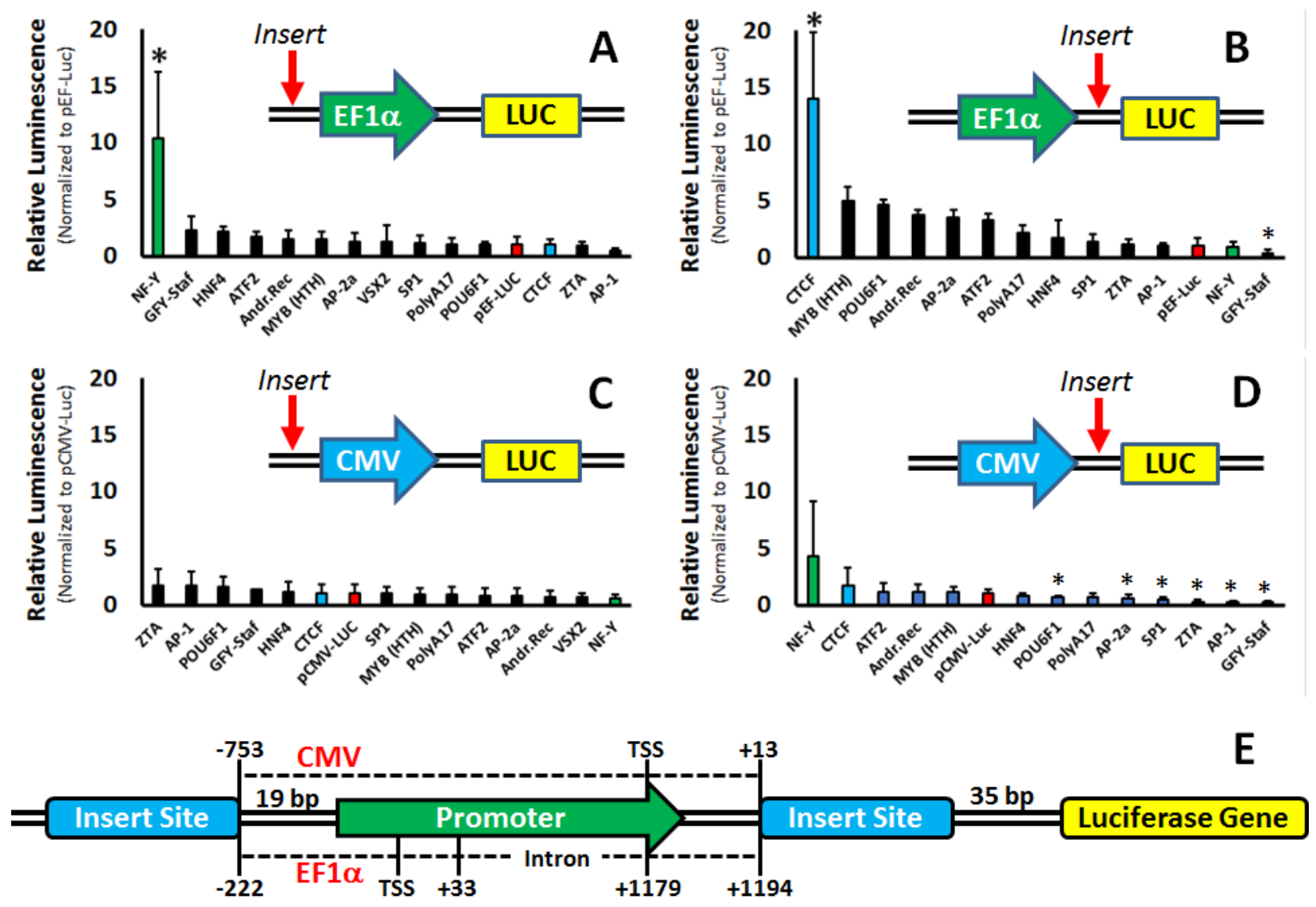

Figure 1: Effects of adding motifs upstream and downstream of the human EF1 $\alpha$ (top panels, A \& B) and viral CMV (bottom panels, C \& D) promoters. The exact location of each insert relative to the transcription start site (TSS) is shown in the bottom panel (E). Experiments were performed in PC-3 (human prostate cancer) cells using branched PEI (8:1 N/P ratio). *significant difference relative to control plasmid ( $\mathrm{p} \leq 0.05$ for $\mathrm{n} \geq 3$ independent experiments). 


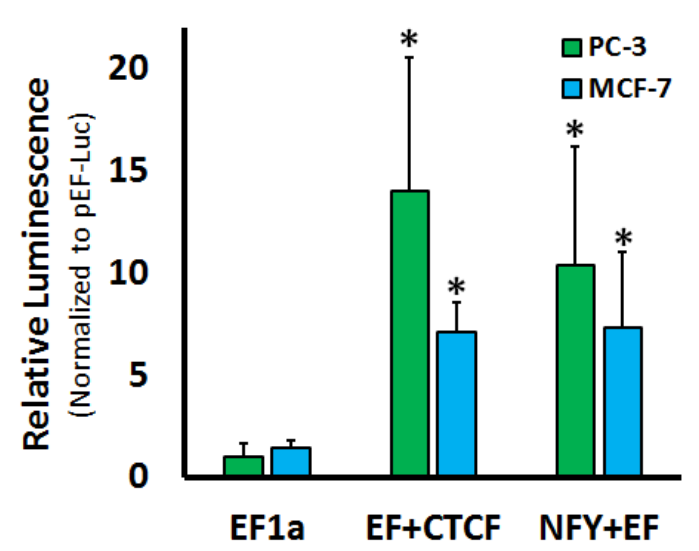

Figure 2 - Effects of CTCF and NF-Y motifs on transgene expression in PC-3 and MCF-7 cells. PC-3 cells were transfected with PEI, while MCF-7 cells were transfected with Lipofectamine. Asterisks $(*)$ indicate significant increases relative to EF1 $\alpha$ ( $\mathrm{p} \leq 0.05$ for $\mathrm{n} \geq 3$ independent experiments).

To determine if the enhancement observed with the NF-Y and CTCF motifs could also be observed with other cell lines and other gene delivery vehicles, we used Lipofectamine to transfect the plasmids into breast cancer cells (MCF-7). As shown in Figure 2, plasmids containing the upstream NF-Y or CTCF motifs significantly enhanced luciferase expression in the MCF-7 cell line, although the magnitude of enhancement was only 7-fold. Similar experiments were also conducted with the Jurkat line of leukemia T cells, but the motifs did not provide any significant enhancement relative to the native EF1 $\alpha$ promoter (data not shown).

\section{Further Investigation of the NF-Y Motif}

Nuclear Transcription Factor Y (NF-Y), also known as CCAAT Binding Factor (CBF) or CCAAT Protein 1 (CP1), is a trimeric protein comprised of three subunits: NF-YA, NF-YB, and NF-YC. ${ }^{39}$ While the NF-YA subunit is responsible for binding the CCAAT motif, the NF-YB and NF-YC subunits contain histone-fold domains (HFDs) that can mimic and replace histones H2A \& H2B, respectively. ${ }^{40}$ NF-Y binding can even occur in relatively inaccessible regions of heterochromatin, thereby allowing other transcription factors and RNA Polymerase to activate transcription of otherwise silenced genes. ${ }^{41} \mathrm{NF}-\mathrm{Y}$ is also associated with several histone modifications (H3K4me3, H3K79me2, and H3K36me3) that activate gene expression. ${ }^{42}$ In addition, the NF-YB subunit can also be post-translationally modified at Lys138 to a ubiquitinated form that resembles H2B Lys120 monoubiquitination, which is associated with transcriptional activation. ${ }^{40}$ Therefore, there are several ways in which adding an NF-Y motif to the EF1 $\alpha$ promoter could provide the observed enhancement of transgene expression.

In the human genome, NF-Y motifs are frequently found in pairs with short spacers of 24-31 bp between each CCAAT box. ${ }^{39,42,43}$ Since the CCAAT motif identified in our initial screen only contains a single CCAAT box, we investigated the effects of inserting tandem CCAAT motifs separated by 24,29 , or 31 bp spacers upstream of the EF1 $\alpha$ promoter. 
Surprisingly, each of the tandem NF-Y motifs significantly decreased transgene expression by $50-60 \%$ relative to the single NF-Y motif (Figure S6).

While tandem NF-Y sites are common in the human genome, some of the individual NF$\mathrm{Y}$ motifs in the CMV promoter are separated by approximately $200 \mathrm{bp}$, which roughly corresponds to the distance between nucleosomes. We next investigated if nucleosomal spacing of NF-Y motifs could further enhance transgene expression by inserting additional NF-Y motifs at other sites in the plasmid (Figure 3). First of all, it is interesting to note that moving the single NF-Y to the ampicillin resistance gene (position -471), inside the EF1 $\alpha$ promoter (position -45), or even past the TSS to the intron within the EF1 $\alpha$ promoter region (position +145 ) provided the same level of expression obtained with the CCAAT motif at its initial location (position -248). In most cases, combining pairs of NF-Y motifs at these locations had no significant effect on transgene expression. Introducing pairs of NF-Y motifs gave no significant increase in transgene expression, although a slight ( 4 -fold) increase in transgene expression seemed to occur when a combination of NF-Y motifs was inserted at -471 and -248 (but the overall difference was not significant).

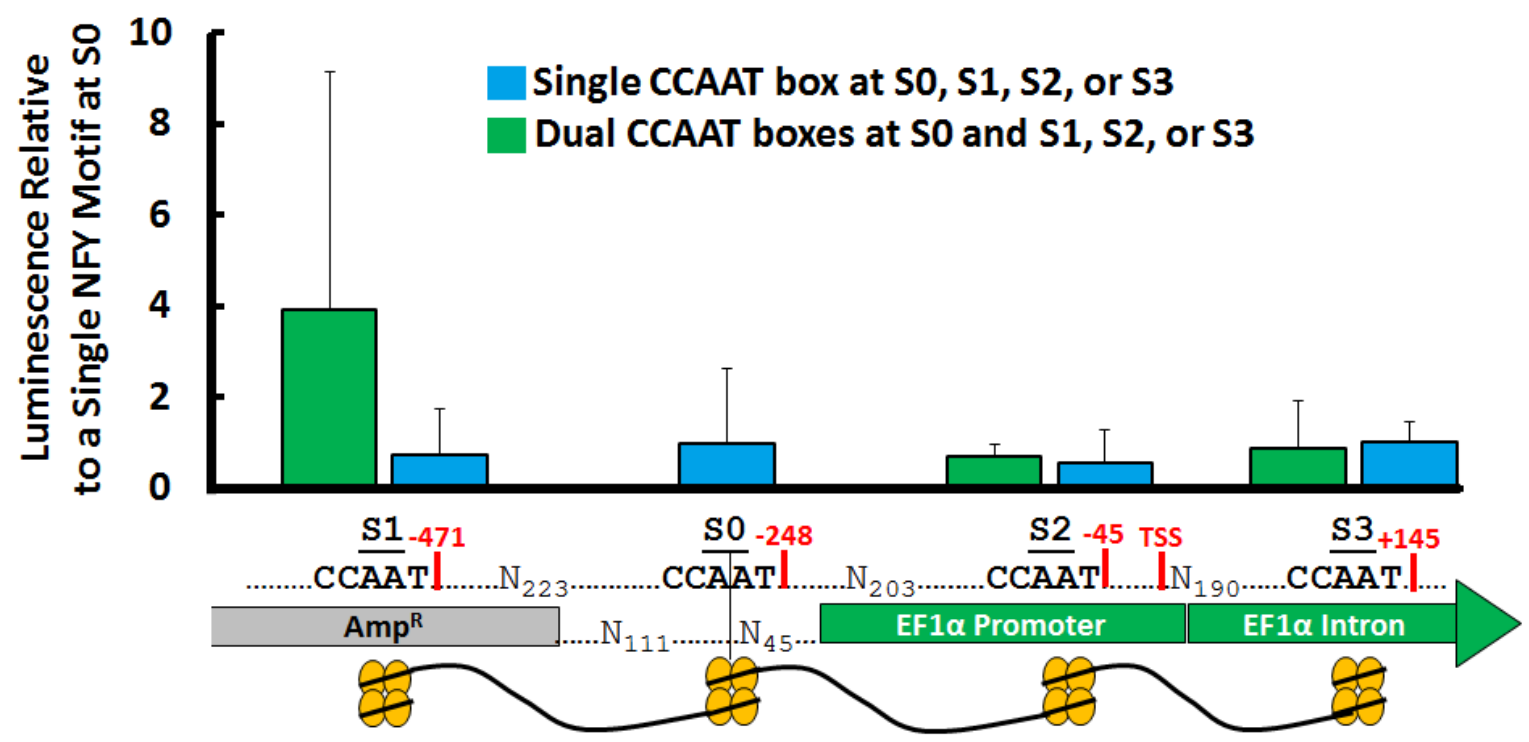

Figure 3 - Effects of single NF-Y motifs and pairs of NF-Y motifs relative to a plasmid with a single NF-Y motif at position -248 relative to the transcription start site (TSS) of the EF1 $\alpha$ promoter. The S0 site is located $19 \mathrm{bp}$ upstream of the EF1 $\alpha$ promoter region and was used for the initial motif screening experiments. The blue bars represent plasmids with single NF-Y motifs inserted at each location, while the green bars represent plasmids with NF-Y motifs present at that position and position -248 .

\section{Further Investigation of the CTCF Motif}

CTCF has a wide range of functions within the genome, including both repression and activation of genes. ${ }^{44}$ Regarding gene activation, the nucleosomes that flank CTCF sites tend to be highly enriched in the histone variant H2A.Z, which flanks nucleosome free regions and is associated with activating histone modifications (e.g., H3K4me3 and H3K4me). ${ }^{45-47}$ The histone variant $\mathrm{H} 2 \mathrm{~A}$.Z itself is multifunctional protein involved both with transcriptional inactivation and 
activation through recruitment of pioneering transcription factors such as FOXA2. ${ }^{48}$ CTCF has also been shown to activate poly(ADP-ribose) polymerase 1 (PARP1) which ADP-ribosylates DNMT1, thus preventing the enzyme from methylating CpG motifs. ${ }^{49,50}$ Recently, CTCF has also been shown to control chromatin conformation by interacting with cohesin. Cohesin, a trimeric protein comprised of SMC1, SMC3, and SCC3 subunits, ${ }^{51}$ does not directly bind to the DNA but instead interacts with CTCF through its SCC3 subunit. ${ }^{52}$ The CTCF/cohesion complex forms loops of chromatin between oppositely oriented CTCF motifs, thereby bringing distal enhancer elements in contact with promoters to increase transcription. ${ }^{53,54}$

Previous studies have shown that CTCF binds DNA using 11 zinc finger domains. ${ }^{55}$ The number of zinc fingers that bind the target DNA and the orientation of the binding site (plus/minus) have also been shown to determine the effects of CTCF binding (e.g., activation or repression of a gene). Since the CTCF motif used in our initial screen only bound 4 of the 11 zinc fingers in the sense (plus) orientation, we attempted to stabilize CTCF binding and potentially increase transgene expression by testing two additional CTCF motifs: (1) an alternative CTCF motif with a slightly different sequence than the initial CTCF motif ${ }^{35}$ and (2) a longer CTCF motif that binds 8 of the $11 \mathrm{CTCF}$ zinc finger domains. ${ }^{55}$ Both of these sequences were tested in the plus and minus orientations as well. Figure 5 shows that the alternative CTCF motif provided significantly decreased transgene expression, but elongating the CTCF motif to bind additional zinc finger domains within CTCF did significantly increase transgene expression 3-fold relative to the initial CTCF motif. Interestingly, however, switching the orientation of the longer CTCF motif from minus to plus caused a significant decrease in transgene expression relative to the initial CTCF motif.

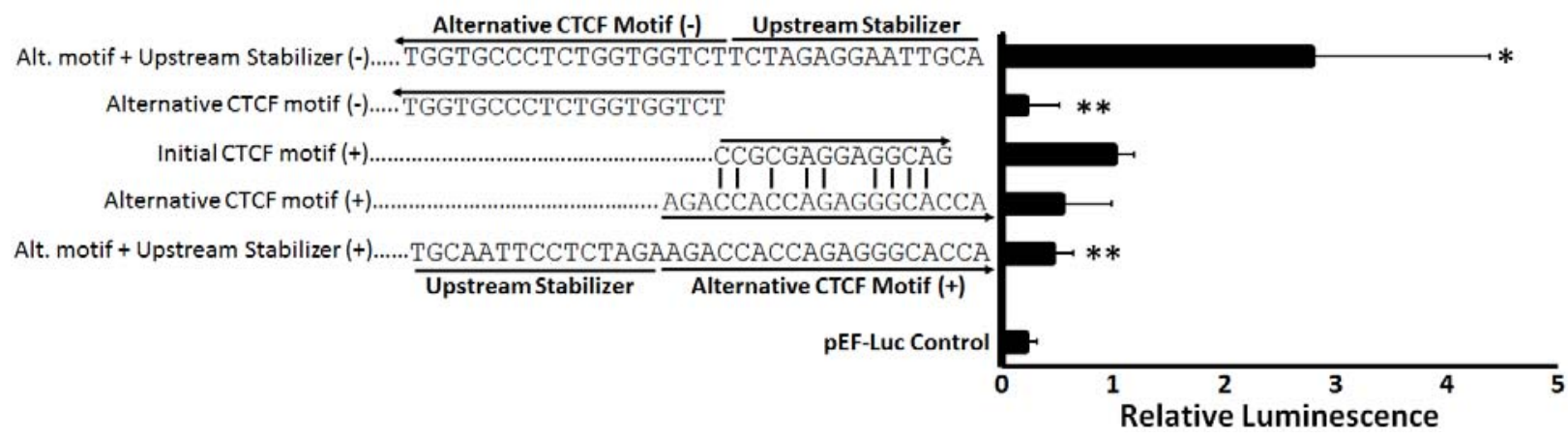

Figure 4: Optimization of the CTCF sequence and its orientation. The initial CTCF motif was used in the screening experiments, while the alternative CTCF motif contains a slightly different sequence that binds 4 the 11 zinc finger domains within CTCF. In addition, an upstream stabilizer sequence was also added to the core CTCF motif to facilitate binding of 4 more zinc finger domains from CTCF. Each of these motifs was inserted downstream of the EF1 $\alpha$ promoter in both the sense (+) and antisense (-) orientation. Single asterisks (*) indicate plasmids with transgene expression/luminescence values significantly higher than plasmids containing the initial CTCF, while double asterisks (**) indicate significant decreases in luminescence ( $\mathrm{p}<0.05, \mathrm{n} \geq 3$ independent experiments). 


\section{Epigenetic Effects of the NF-Y and CTCF Motifs}

ChIP assays were performed to confirm that the enhancement observed with the NF-Y and CTCF motifs was indeed due to NF-Y or CTCF binding the plasmid. As shown in Figure $4 \mathrm{a}$, the amount of NF-Y bound to the EF1 $\alpha$ promoter region significantly increased upon addition of the NF-Y motif to the EF1 $\alpha$ promoter. Likewise, inserting the expanded CTCF motif with a stabilizer domain (see Figure 4) downstream of the EF1 $\alpha$ promoter also exhibited a significant increase in CTCF binding relative to the native EF1 $\alpha$ promoter. In both cases, the \%input values for the NF-Y and CTCF samples were also significantly higher than the \%input values for their corresponding negative control IgG. Therefore, it appears that the enhancement observed with these motifs is due to the recruitment of NF-Y or CTCF to the plasmid. However, the ChIP experiments did not show any changes in histone $\mathrm{H} 3$ binding or local $\mathrm{H} 3 \mathrm{~K} 9$ acetylation, so the change in transgene expression may be due to some other type of histone modification or the recruitment of additional transcription factors/proteins by NF-Y or CTCF.
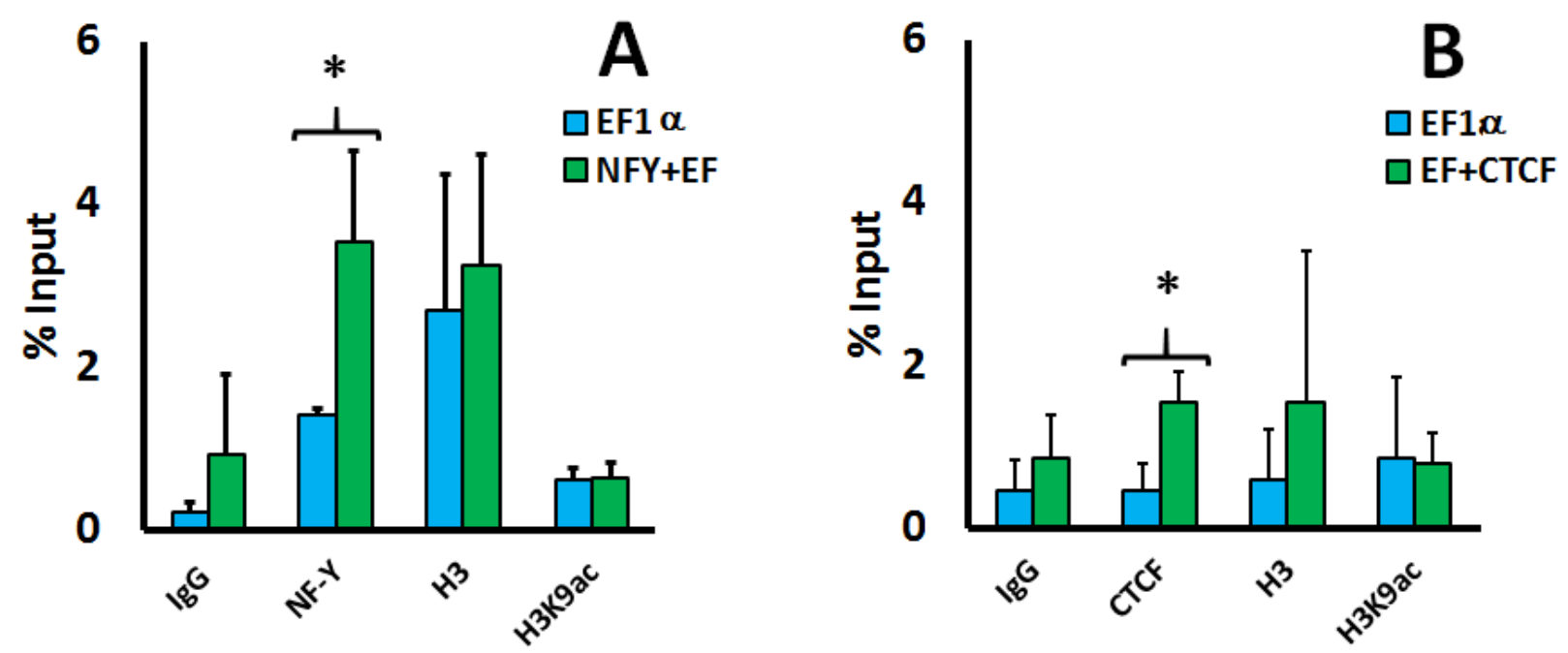

Figure 5 -Transiently transfected plasmids with the NF-Y motif (left panel, A) or the CTCF motif (right panel, B) were tested for NF-Y, CTCF, H3, and H3K9ac binding relative to the native EF1 $\alpha$ promoter in PC-3 cells. Asterisks (*) indicate a significant increase in occupancy of the target protein relative to the native promoter $(\mathrm{EF} 1 \alpha)$.

\section{Nuclear Uptake Effects}

Since it is possible that the enhancement observed with each motif may be due to NF-Y or CTCF binding the plasmid in the cytoplasm and then increasing its rate of nuclear transport, we also calculated the fold-change in nuclear copy number for the plasmids with the motifs (plasmid copy numbers were normalized to the housekeeping gene RPL30). Interestingly, Figure 6 shows that the addition of the NF-Y motif significantly decreases the plasmid copy number by approximately $20 \%$ (relative to a plasmid containing the native EF1 $\alpha$ promoter). In contrast, the CTCF motif had no significant effect on plasmid copy number. Therefore, it appears that the enhancement provided by the NF-Y and CTCF motifs are not due to an increase in nuclear copy number. 


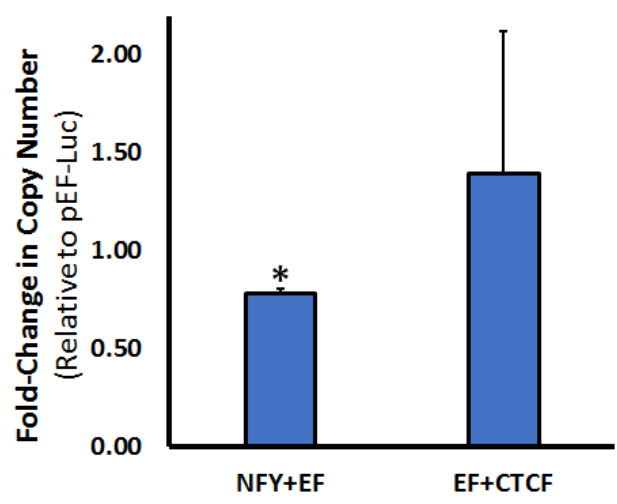

Figure 6 - Fold-change in copy number of plasmids with the NF-Y motif $(\mathrm{NFY}+\mathrm{EF})$ or the $\mathrm{CTCF}$ motif $(\mathrm{EF}+\mathrm{CTCF})$ relative to a plasmid with the native EF1 $\alpha$ promoter. Copy numbers were normalized to the housekeeping gene RPL30 (data not shown). Asterisks (*) indicate a significant decrease in nuclear copy number relative to the plasmid with the native promoter $(\mathrm{EF} 1 \alpha)$.

\section{Conclusion}

Previous studies have shown that foreign transgenes are susceptible to epigenetic silencing, but our results show that transgene expression can be significantly increased by modifying the EF1 $\alpha$ promoter to recruit pioneering transcription factors like NF-Y and chromatin remodeling enzymes like CTCF. The exact mechanism by which NF-Y and CTCF increase transgene expression is currently unknown, but both of these proteins are known to significantly remodel chromatin by replacing histones (NF-Y) or forming chromatin loops that insulate specific loci (CTCF). Therefore, the conformation of the plasmid chromatin may be a particularly important epigenetic factor that regulates transgene expression.

\section{Acknowledgements}

This work was supported by NSF CBET Grant 1403214. The authors have no conflicts of interest related to this work.

\section{References}

1. Grewal SIS, Moazed D. Heterochromatin and epigenetic control of gene expression. Science. 2003;301(5634):798-802.

2. Margueron R, Trojer P, Reinberg D. The key to development: interpreting the histone code? Curr Opin Genet Dev. 2005;15(2):163-176.

3. Kim J, Kim H. Recruitment and Biological Consequences of Histone Modification of H3K27me3 and H3K9me3. ILAR J. 2012;53(3-4):232-239.

4. Jähner D, Stuhlmann H, Stewart CL, et al. De novo methylation and expression of retroviral genomes during mouse embryogenesis. Nature. 1982;298(5875):623-628. 
5. Brooks AR, Harkins RN, Wang P, Qian HS, Liu P, Rubanyi GM. Transcriptional silencing is associated with extensive methylation of the CMV promoter following adenoviral gene delivery to muscle. J Gene Med. 2004;6(4):395-404.

6. Ellis J. Silencing and Variegation of Gammaretrovirus and Lentivirus Vectors. Hum Gene Ther. 2005;16(11):1241-1246.

7. Ross PJ, Kennedy MA, Parks RJ. Host cell detection of noncoding stuffer DNA contained in helper-dependent adenovirus vectors leads to epigenetic repression of transgene expression. J Virol. 2009;83(17):8409-8417.

8. Suzuki M, Cerullo V, Bertin TK, et al. MyD88-dependent silencing of transgene expression during the innate and adaptive immune response to helper-dependent adenovirus. Hum Gene Ther. 2010;21(3):325-336.

9. Gracey Maniar LE, Maniar JM, Chen Z-Y, Lu J, Fire AZ, Kay MA. Minicircle DNA vectors achieve sustained expression reflected by active chromatin and transcriptional level. Mol Ther. 2013;21(1):131-138.

10. Riu E, Chen Z-Y, Xu H, He C-Y, Kay MA. Histone modifications are associated with the persistence or silencing of vector-mediated transgene expression in vivo. Mol Ther. 2007;15(7):1348-1355.

11. Chen ZY, He CY, Meuse L, Kay MA. Silencing of episomal transgene expression by plasmid bacterial DNA elements in vivo. Gene Ther. 2004;11(10):856-864.

12. Huang Y, Stewart TM, Wu Y, et al. Novel oligoamine analogues inhibit lysine-specific demethylase 1 and induce reexpression of epigenetically silenced genes. Clin Cancer Res. 2009;15(23):7217-7228.

13. Wang J, Hevi S, Kurash JK, et al. The lysine demethylase LSD1 (KDM1) is required for maintenance of global DNA methylation. Nat Genet. 2009;41(1):125-129.

14. Santi D V, Garrett CE, Barr PJ. On the mechanism of inhibition of DNA-cytosine methyltransferases by cytosine analogs. Cell. 1983;33(1):9-10.

15. Cheng JC, Matsen CB, Gonzales FA, et al. Inhibition of DNA methylation and reactivation of silenced genes by zebularine. J Natl Cancer Inst. 2003;95(5):399-409.

16. Elmer JJ, Christensen MD, Barua S, Lehrman J, Haynes KA, Rege K. The histone deacetylase inhibitor Entinostat enhances polymer-mediated transgene expression in cancer cell lines. Biotechnol Bioeng. 2016;113(6):1345-1356.

17. Barua S, Rege K. The influence of mediators of intracellular trafficking on transgene expression efficacy of polymer-plasmid DNA complexes. Biomaterials.

2010;31(22):5894-5902.

18. Gaetano C, Catalano A, Palumbo R, et al. Transcriptionally active drugs improve adenovirus vector performance in vitro and in vivo. Gene Ther. 2000;7(19):1624-1630.

19. Kim Y-E, Park J-A, Park S-K, Kang H-B, Kwon H-J, Lee Y. Enhancement of Transgene Expression by HDAC Inhibitors in Mouse Embryonic Stem Cells. Dev Reprod. 2013;17(4):379-387.

20. Ho YK, Zhou LH, Tam KC, Too HP. Enhanced non-viral gene delivery by coordinated endosomal release and inhibition of $\beta$-tubulin deactylase. Nucleic Acids Res. 2017;45(6):e38.

21. Marks PA, Rifkind RA, Richon VM, Breslow R, Miller T, Kelly WK. Histone deacetylases and cancer: causes and therapies. Nat Rev Cancer, Publ online 01 December 2001; | doil01038/35106079. 2001;1(3):194.

22. Marks PA, Dokmanovic M. Histone deacetylase inhibitors: discovery and development as 
anticancer agents. Expert Opin Investig Drugs. 2005;14(12):1497-1511.

23. Nicolson SC, Li C, Hirsch ML, Setola V, Samulski RJ. Identification and Validation of Small Molecules That Enhance Recombinant Adeno-associated Virus Transduction following High-Throughput Screens. J Virol. 2016;90(16):7019-7031.

24. Lu J, Zhang F, Kay MA. A mini-intronic plasmid (MIP): a novel robust transgene expression vector in vivo and in vitro. Mol Ther. 2013;21(5):954-963.

25. Osborn MJ, McElmurry RT, Lees CJ, et al. Minicircle DNA-based gene therapy coupled with immune modulation permits long-term expression of $\alpha$-L-iduronidase in mice with mucopolysaccharidosis type I. Mol Ther. 2011;19(3):450-460.

26. Vaissière T, Sawan C, Herceg Z. Epigenetic interplay between histone modifications and DNA methylation in gene silencing. Mutat Res Mutat Res. 2008;659(1-2):40-48.

27. Klose RJ, Bird AP. Genomic DNA methylation: the mark and its mediators. Trends Biochem Sci. 2006;31(2):89-97.

28. Moritz B, Becker PB, Göpfert U. CMV promoter mutants with a reduced propensity to productivity loss in CHO cells. Sci Rep. 2015;5(1):16952.

29. Chen CJ, Deng Z, Kim AY, Blobel GA, Lieberman PM. Stimulation of CREB binding protein nucleosomal histone acetyltransferase activity by a class of transcriptional activators. Mol Cell Biol. 2001;21(2):476-487.

30. Montminy MR. Identification of a Cyclic-AMP-Responsive Element within the Rat Somatostatin Gene. Proc Natl Acad Sci. 1986;83(18):6682-6686.

31. Kriwacki RW, Schultz SC, Steitz TA, Caradonna JP. Sequence-specific recognition of DNA by zinc-finger peptides derived from the transcription factor Sp1. Proc Natl Acad Sci. 1992;89(20):9759-9763.

32. Fang B, Mane-Padros D, Bolotin E, Jiang T, Sladek FM. Identification of a binding motif specific to HNF4 by comparative analysis of multiple nuclear receptors. Nucleic Acids Res. 2012;40(12):5343-5356.

33. Lee D, Karchin R, Beer MA. Discriminative prediction of mammalian enhancers from DNA sequence. Genome Res. 2011;21(12):2167-2180.

34. Hong CP, Choe MK, Roh T-Y. Characterization of Chromatin Structure-associated Histone Modifications in Breast Cancer Cells. Genomics Inform. 2012;10(3):145-152.

35. Kim TH, Abdullaev ZK, Smith AD, et al. Analysis of the vertebrate insulator protein CTCF-binding sites in the human genome. Cell. 2007;128(6):1231-1245.

36. Roche PJ, Hoare SA, Parker MG. A consensus DNA-binding site for the androgen receptor. Mol Endocrinol. 1992;6(12):2229-2235.

37. Raveh-Sadka T, Levo M, Shabi U, et al. Manipulating nucleosome disfavoring sequences allows fine-tune regulation of gene expression in yeast. Nat Genet. 2012;44(7):743-750.

38. Joseph R, Orlov YL, Huss M, et al. Integrative model of genomic factors for determining binding site selection by estrogen receptor- $\alpha$. Mol Syst Biol. 2010;6(1):456.

39. Dolfini D, Zambelli F, Pavesi G, Mantovani R. A perspective of promoter architecture from the CCAAT box. Cell Cycle. 2009;8(24):4127-4137.

40. Nardini M, Gnesutta N, Donati G, et al. Sequence-Specific Transcription Factor NF-Y Displays Histone-like DNA Binding and H2B-like Ubiquitination. Cell. 2013;152(12):132-143.

41. Fleming JD, Pavesi G, Benatti P, Imbriano C, Mantovani R, Struhl K. NF-Y coassociates with FOS at promoters, enhancers, repetitive elements, and inactive chromatin regions, and is stereo-positioned with growth-controlling transcription factors. Genome Res. 
2013;23(8):1195-1209.

42. Dolfini D, Gatta R, Mantovani R. NF-Y and the transcriptional activation of CCAAT promoters. Crit Rev Biochem Mol Biol. 2012;47(1):29-49.

43. Dolfini D, Zambelli F, Pedrazzoli M, Mantovani R, Pavesi G. A high definition look at the NF-Y regulome reveals genome-wide associations with selected transcription factors. Nucleic Acids Res. 2016;44(10):4684-4702.

44. Kim S, Yu N-K, Kaang B-K. CTCF as a multifunctional protein in genome regulation and gene expression. Exp Mol Med. 2015;47(6):e166.

45. Fu Y, Sinha M, Peterson CL, Weng Z. The Insulator Binding Protein CTCF Positions 20 Nucleosomes around Its Binding Sites across the Human Genome. van Steensel B, ed. PLoS Genet. 2008;4(7):e1000138.

46. Raisner RM, Hartley PD, Meneghini MD, et al. Histone variant H2A.Z marks the 5' ends of both active and inactive genes in euchromatin. Cell. 2005;123(2):233-248.

47. Barski A, Cuddapah S, Cui K, et al. High-resolution profiling of histone methylations in the human genome. Cell. 2007;129(4):823-837.

48. Subramanian V, Fields PA, Boyer LA. H2A.Z: a molecular rheostat for transcriptional control. F1000Prime Rep. 2015;7:1.

49. Zampieri M, Guastafierro T, Calabrese R, et al. ADP-ribose polymers localized on CtcfParp1-Dnmt1 complex prevent methylation of Ctcf target sites. Biochem J. 2012;441(2):645-652.

50. Guastafierro T, Cecchinelli B, Zampieri M, et al. CCCTC-binding Factor Activates PARP-1 Affecting DNA Methylation Machinery. J Biol Chem. 2008;283(32):2187321880.

51. Nasmyth K, Haering CH. Cohesin: Its Roles and Mechanisms. Annu Rev Genet. 2009;43(1):525-558.

52. Nanbo A, Sugden A, Sugden B, Kissil JL, Bartolomei MS, Lieberman PM. The coupling of synthesis and partitioning of EBV's plasmid replicon is revealed in live cells. EMBO J. 2007;26(19):4252-4262.

53. Kagey MH, Newman JJ, Bilodeau S, et al. Mediator and cohesin connect gene expression and chromatin architecture. Nature. 2010;467(7314):430-435.

54. Faure AJ, Schmidt D, Watt S, et al. Cohesin regulates tissue-specific expression by stabilizing highly occupied cis-regulatory modules. Genome Res. 2012;22(11):2163-2175.

55. Nakahashi H, Kieffer Kwon K-R, Resch W, et al. A genome-wide map of CTCF multivalency redefines the CTCF code. Cell Rep. 2013;3(5):1678-1689. 


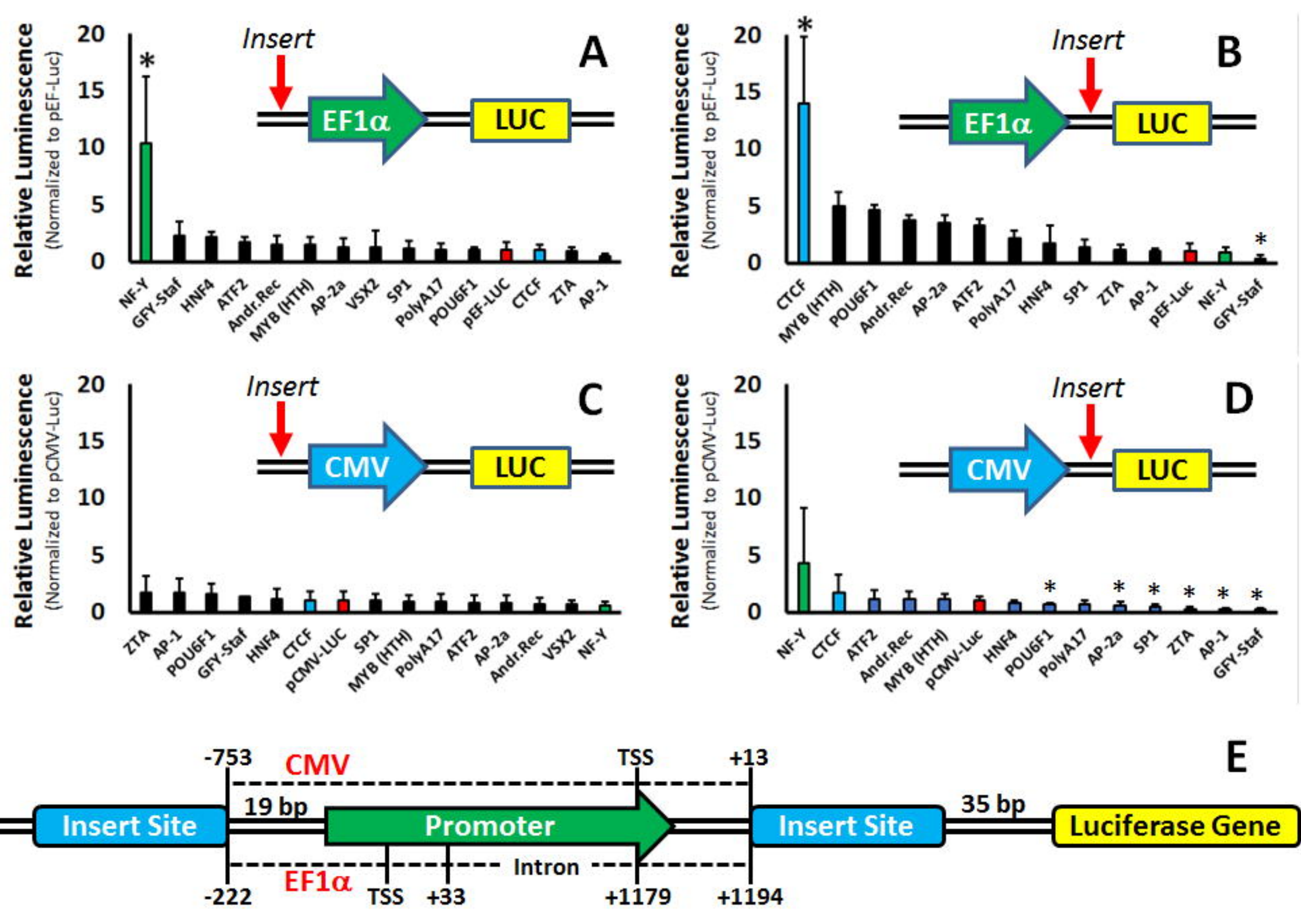




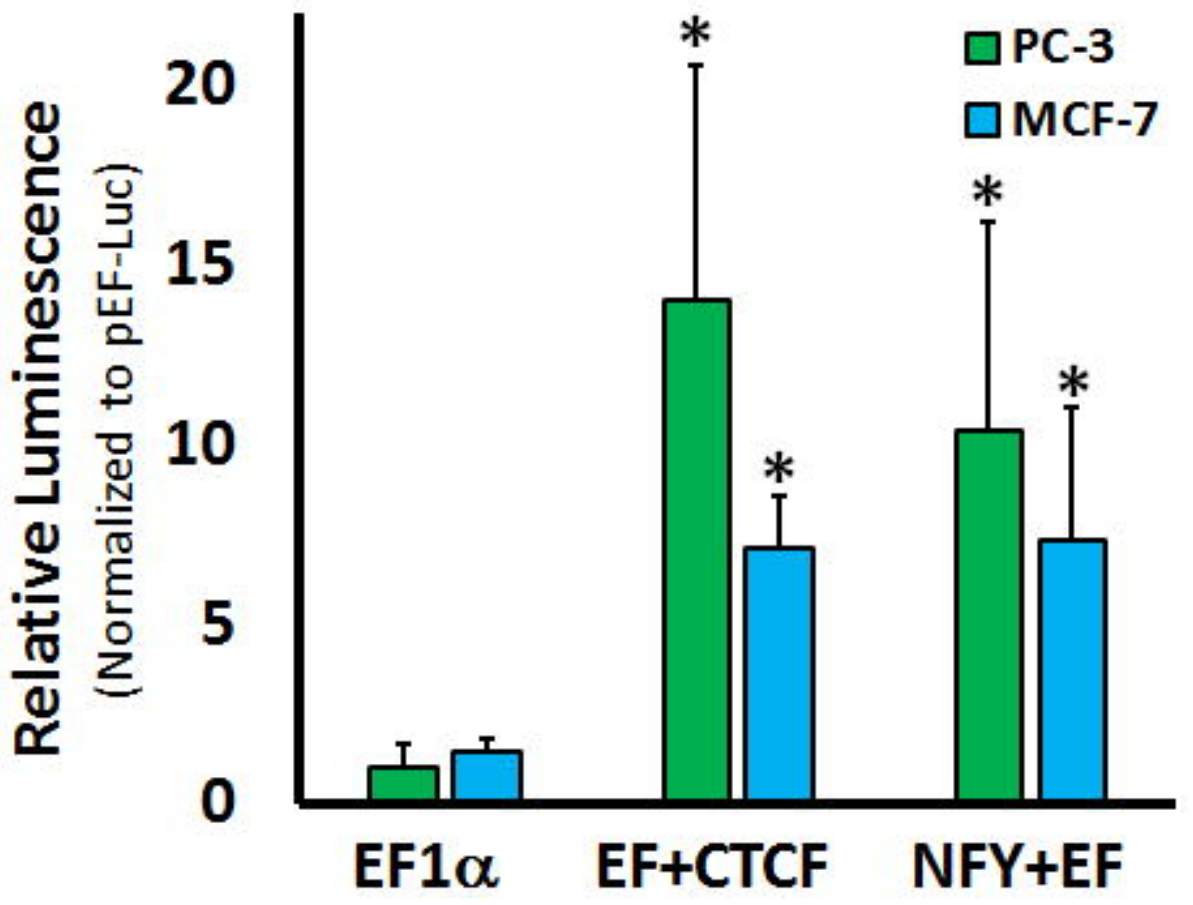




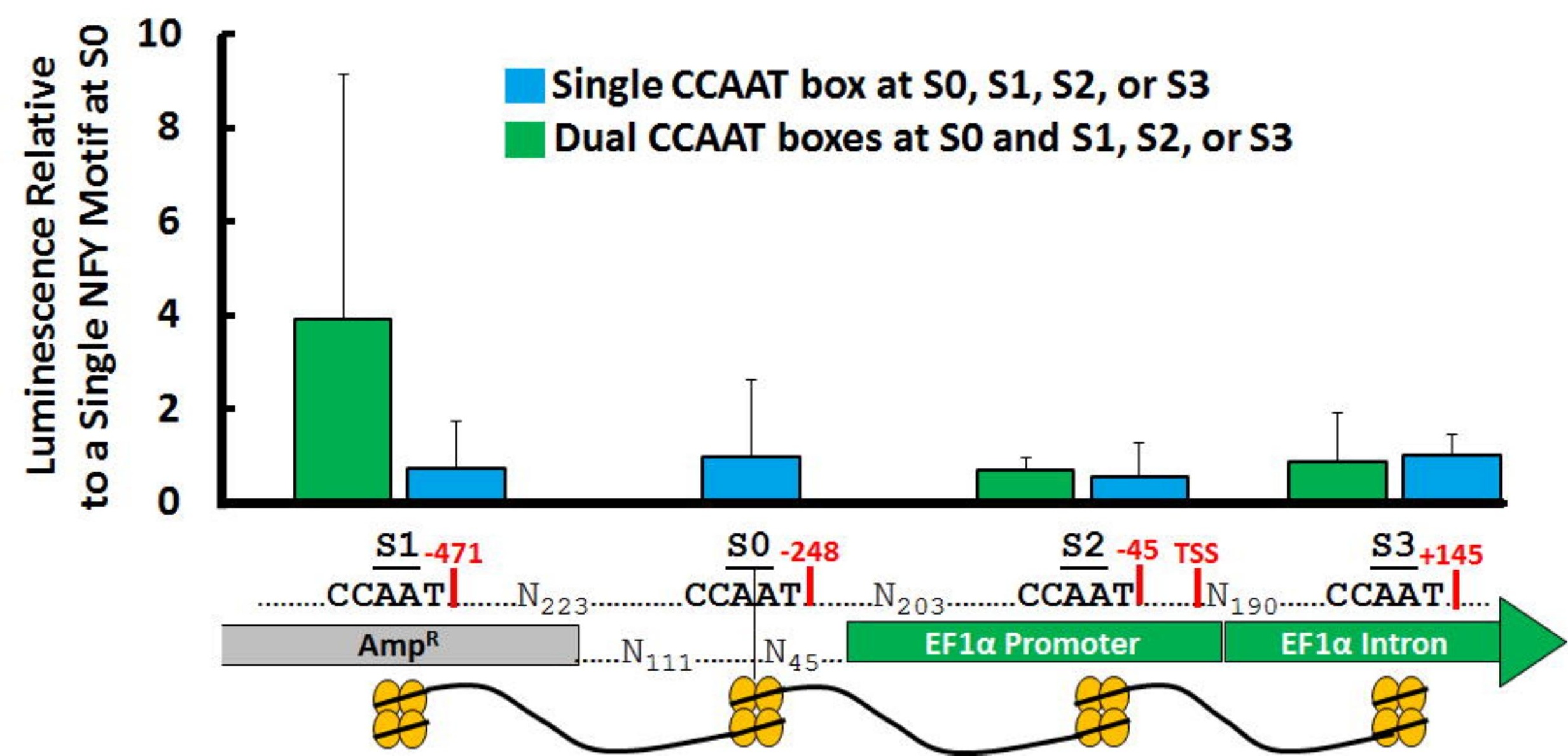


Alt. motif + Upstream Stabilizer (-)..... TGGTGCCCTCTGGTGGTCTTCTAGAGGAATTGCA

Alternative CTCF motif (-)..... Initial CTCF motif (+)...

Alternative CTCF motif $(+)$... $\overrightarrow{\mathrm{CCGCGAGGAGGCAG}}$ I I I । III $\stackrel{\text { AGACCACCAGAGGGCACCA }}{\longrightarrow}$

Alt. motif + Upstream Stabilizer (+)......TGCAATTCCTCTAGAAGACCACCAGAGGGCACCA

Upstream Stabilizer $\quad$ Alternative CTCF Motif (+)

pEF-Luc Control 


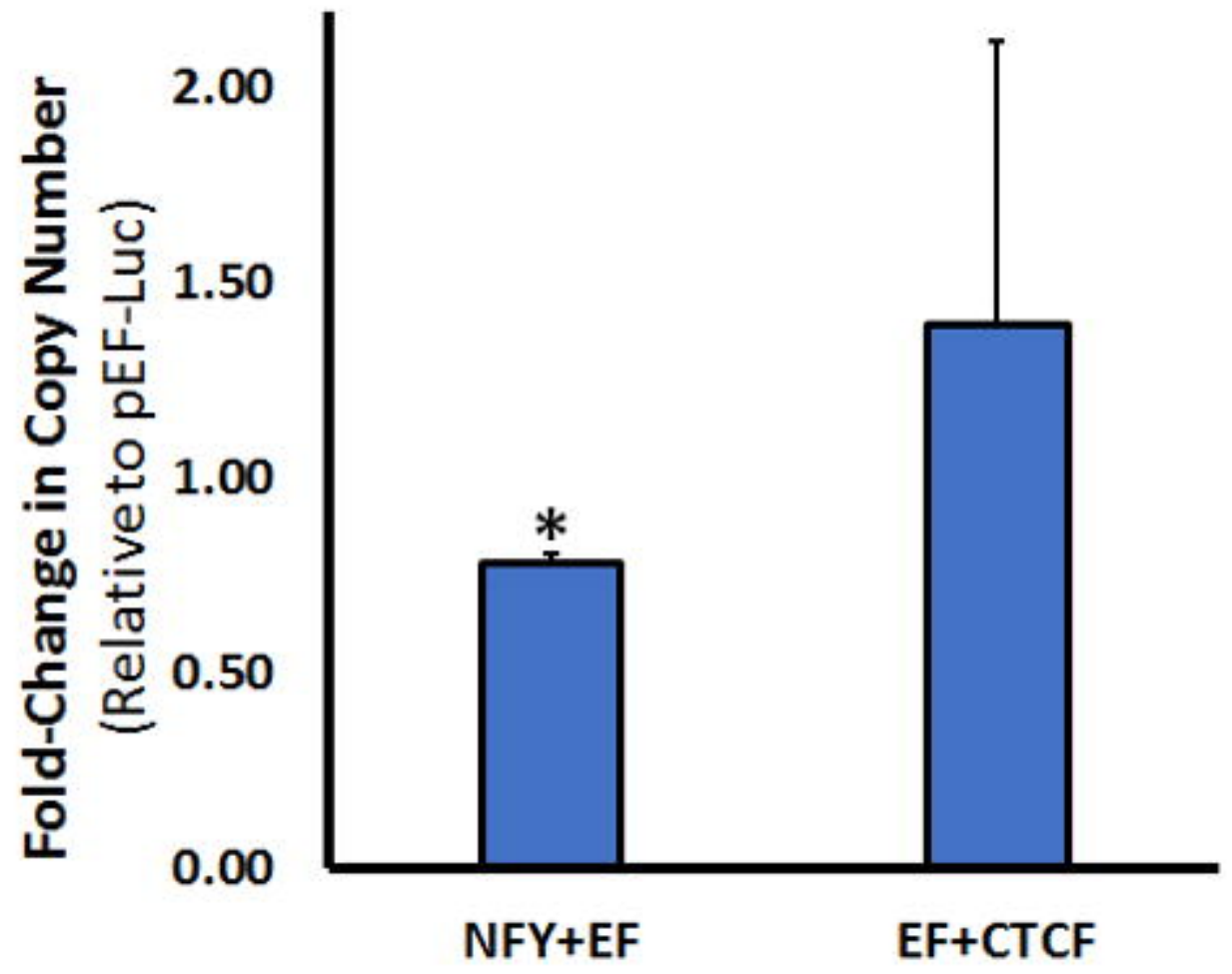

